\title{
Coalescence of drops on the free-surface of a liquid pool at elevated temperatures
}

Cite as: Phys. Fluids 32, 052103 (2020); https://doi.org/10.1063/5.0007402

Submitted: 15 March 2020 . Accepted: 23 April 2020 . Published Online: 06 May 2020

Pavan Kumar Kirar, Kathryn Alvarenga, (D) Pankaj Kolhe, (D) Gautam Biswas, and (D) Kirti Chandra Sahu

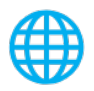

\section{ARTICLES YOU MAY BE INTERESTED IN}

Coalescence dynamics of a droplet on a sessile droplet

Physics of Fluids 32, 012104 (2020); https://doi.org/10.1063/1.5129901

Impact of an air-in-liquid compound drop onto a liquid surface

Physics of Fluids 32, 041705 (2020); https://doi.org/10.1063/5.0005702

Splashing dynamics of a drop impact onto a deep liquid pool with moving film interface Physics of Fluids 32, 012102 (2020); https://doi.org/10.1063/1.5131637

\section{Physics of Fluids}

SPECIAL TOPIC: Tribute to

Frank M. White on his 88th Anniversary 


\title{
Coalescence of drops on the free-surface of a liquid pool at elevated temperatures
}

\author{
Cite as: Phys. Fluids 32, 052103 (2020); doi: 10.1063/5.0007402 \\ Submitted: 15 March 2020 - Accepted: 23 April 2020 • \\ Published Online: 6 May 2020
}

\section{(1) 屯 (1)}

Pavan Kumar Kirar, ${ }^{1}$ (D) Kathryn Alvarenga, ${ }^{2}$ Pankaj Kolhe, ${ }^{3}$ (D) Gautam Biswas, ${ }^{4}$ (D) and Kirti Chandra Sahu ${ }^{1, a)}$ (D)

\begin{abstract}
AFFILIATIONS
${ }^{1}$ Department of Chemical Engineering, Indian Institute of Technology Hyderabad, Sangareddy 502 285, Telangana, India

${ }^{2}$ Department of Aerospace Engineering, Texas A\&M University, College Station, Texas 77843-3141, USA

${ }^{3}$ Department of Mechanical and Aerospace Engineering, Indian Institute of Technology Hyderabad,

Sangareddy 502 285, Telangana, India

4Department of Mechanical Engineering, Indian Institute of Technology Kanpur, Kanpur 208016, India
\end{abstract}

a) Author to whom correspondence should be addressed: ksahu@che.iith.ac.in

\begin{abstract}
The coalescence dynamics of ethanol drops injected from a needle on the free-surface of an ethanol pool maintained at a higher temperature than the drop is experimentally studied using a high-speed imaging system. The drop is always kept at $25^{\circ} \mathrm{C}$, and the temperature of the ethanol pool is varied using a heater. The coalescence behavior depends on the size of the drop, the height of the needle tip from the freesurface, and the temperature of the ethanol pool. A parametric study is carried out by varying these parameters. The drop exhibits a residence period at low impact velocity, when it floats on the free-surface before the coalescence begins. Subsequently, the complete coalescence and partial coalescence dynamics are observed for different sets of parameters considered. It is found that increasing the temperature of the ethanol pool reduces the residence time of the drop. This phenomenon is explained by analyzing the forces acting on the drop and the capillary waves generated due to the temperature gradient between the drop and the ethanol pool. During partial coalescence, we also observed that the diameter of the daughter droplet decreases as the size of the primary drop and pool temperature are increased. As expected, due to the gravity effect, increasing the size of the drop also decreases the residence time. A regime map designating the complete coalescence and partial coalescence dynamics is plotted in the pool temperature and drop impact height space.
\end{abstract}

Published under license by AIP Publishing. https://doi.org/10.1063/5.0007402

\section{NOMENCLATURE}

\section{Symbol}

A surface area of the curved surface

$D_{p} \quad$ diameter of the primary drop

$D_{s} \quad$ diameter of the daughter droplet

$F_{b} \quad$ buoyancy force

$F_{l} \quad$ surface tension force

$h$ height of the bottom of the drop from the ethanol-air interface

$H$ height of the needle tip from the ethanol-air interface

$\mathrm{Ma}$ marangoni number

$r_{c} \quad$ radius of the curved surface

$t_{c} \quad$ time scale of thermal diffusion

$t_{r} \quad$ residence time of the drop

$T_{s}$ temperature at the free-surface of an ethanol pool
$U \quad$ impact velocity of the drop

$W \quad$ weight of the drop

$\alpha \quad$ thermal diffusivity of ethanol

$\Delta T$ temperature difference between the drop and pool

$\mu_{l} \quad$ dynamic viscosity of ethanol

$v \quad$ kinematic viscosity of air

$\rho_{a} \quad$ density of air

$\rho_{l} \quad$ density of ethanol

$\sigma \quad$ surface tension

$\tau$ dimensionless time, $t / t_{c}$

$\tau_{r} \quad$ dimensionless residence time, $t_{r} / t_{c}$

\section{INTRODUCTION}

The impact of a drop on the solid or free-surface of a liquid pool has fascinated researchers for many decades because of its 
importance in a wide range of applications, including microfluidics, combustion, coatings, inkjet printing, soil erosion, or air trapping on the sea surface, to name a few (see, for instance, Refs. 1-5). Drop coalescence on a heated liquid pool can also be observed in several practical applications, such as bioprinting, oil industry, production of nanoparticles, and microfluidic technologies. ${ }^{6-9}$ As in the present study, we focus on the dynamics of a drop slowly placed on the freesurface of a liquid pool maintained at different temperatures, we only review the literature concerned with the collision of the drop with a liquid-air interface. A drop falling in a liquid pool undergoes various regimes as the impact velocity increases, such as spreading, partial coalescence, and splashing. ${ }^{10}$ Despite a large volume of work conducted for the isothermal system (when the drop and the liquid pool are at the same temperature; see, for instance, Refs. 11-18), the non-isothermal system (when the drop and the liquid pool are at different temperatures and thermocapillary stresses can play an important role; see Ref. 19) has received far less attention in the literature.

When a small liquid drop comes into contact with a free-surface of a liquid pool in an isothermal environment, the drop undergoes either spreading as it drains completely inside the pool or undergoes partial coalescence dynamics as a satellite drop pinches off depending on its impacting velocity and size, as well as the fluid properties of the liquids. ${ }^{20}$ At low impact velocity, the primary drop injected from a needle floats on the free-surface while the trapped air between the drop and the free-surface drains out. In reaction, the air film of decreasing thickness generates a lubrication force that balances the droplet weight. This period when drop floats on the surface is termed the "residence time."14,21,22 Subsequently, the drop comes in contact with the free-surface at a point, and due to high capillary pressure, it undergoes rapid spreading. This in turn generates upward moving capillary waves that form a liquid column. At this stage, the surface tension $(\sigma)$ squeezed the bottom of the liquid column, which experiences a competition between the vertical and horizontal collapses due to upward moving capillary waves and the surface tension force, respectively. Under certain conditions, the horizontal collapse overcomes the vertical collapse, and the liquid column is detached from free-surface as a daughter drop of diameter about half of the primary drop. ${ }^{14,17}$ The daughter drops completely drain in the liquid pool, under a coalescence cascading cycle. Originally, partial coalescence was thought to be a result of an inviscid instability, ${ }^{11}$ but later it was found to be regulated mainly by gravity, viscosity, and interfacial stress. ${ }^{12,14}$ Couder et al. ${ }^{23}$ have demonstrated that with a silicone oil of kinematic viscosity $500 \mathrm{cSt}$, the critical acceleration of bouncing drop increases as the square of the frequency of oscillation of the surface. We note a similarity with the inelastic bouncing ball model since small drops deform the bath weakly. All the above-mentioned studies are conducted in an isothermal environment. Considering the Marangoni flow resulting from the surfactant gradient, Haldar et al. ${ }^{24}$ reported that the number of coalescence cascading cycles decreases as the surfactant concentration increases.

In non-isothermal configuration, few researchers ${ }^{25-27}$ investigated experimentally the interaction of two drops maintained at different temperatures and found that the drops exhibit noncoalescence behavior due to the presence of thermally induced Marangoni stresses above a critical temperature difference. This finding was also later verified in Refs. 28 and 29 via numerical simulations. Most relevant here are the previous studies in Refs. 19 and 30 on the coalescence of a drop on the free-surface of a pool of the same liquid maintained at a higher temperature than the drop. They observed that the drop floats longer by increasing the temperature difference between the drop and pool $(\Delta T)$. Considering silicone oils with different viscosities as the working fluids, Geri et al. ${ }^{19}$ demonstrated that the residence time $\left(t_{r}\right)$ scales as $\Delta T^{2 / 3}$. This phenomenon can be explained as follows, since the increase in residence time is due to the thermally induced Marangoni flow inside the drop, which causes the air trapped between the drop and the pool to move inward instead of draining outward.

The Marangoni number $(M a)$ is a dimensionless number that characterizes the thermal convection owing to the surface tension gradient, ${ }^{31-33}$

$$
M a=\left(\frac{d \sigma}{d T}\right) \frac{\Delta T D_{p}}{\mu_{l} \alpha},
$$

where $D_{p}$ is the diameter of the primary drop, $\mu_{l}$ is the dynamic viscosity, and $\alpha$ is the thermal diffusivity of the liquid. Increasing $\Delta T$ increases the Marangoni number, which enhances the internal circulation in the upward direction inside the drop. In order to balance this flow, the trapped air compressed inward, thereby maintaining the air cushion between the drop and the pool. However, for the Marangoni convection to develop, the residence time should be higher than the time scale of thermal diffusion $\left(t_{c}=D_{p}{ }^{2} / \alpha\right)$. This is the basic difference between the present study and the previous investigations, ${ }^{30,19}$ which provided significant time for the development of Marangoni convection by anchoring the drop between the needle and free-surface, and the Faraday instabilities that allow the drop to float on the free-surface can also be observed. In contrast, in our experiments, as we allow the drop to fall freely from the needle onto the free-surface of the pool, the residence time is quite low as compared to the diffusion time scale. Ethanol is used as a working fluid. In addition, the size of the tank used in our study is sufficiently large, so that the coalescence dynamics is free from the reflected waves from the boundaries.

In the present study, we revisit the coalescence dynamics of an ethanol drop (always at $25^{\circ} \mathrm{C}$ ) slowly placed on the free-surface of an ethanol pool maintained at different temperatures $\left(T_{s}\right)$ by conducting experiments using a high-speed camera. A parametric study is carried out by varying the diameter of the primary drop $\left(D_{p}\right)$ released from a dispensing needle and the height of the needle tip from the ethanol-air interface $(H)$ at different values of $T_{s}$. We observe complete coalescence without the formation of daughter droplet and partial coalescence depending on the parameters considered. A regime map designating the complete coalescence and partial coalescence dynamics is also plotted in the $T_{s}-H$ space.

The rest of the paper is arranged as follows. The details of the experimental setup and procedure followed are given in Sec. II. The results obtained from our study are presented in Sec. III, and the concluding remark is provided in Sec. IV.

\section{EXPERIMENTAL SETUP AND PROCEDURE}

We investigate experimentally the coalescence dynamics of ethanol drops that are slowly placed on the ethanol-air interface in a container (inner size $48 \times 48 \times 48 \mathrm{~mm}^{3}$ ) filled with pure ethanol 
up to $15 \mathrm{~mm}$. The ethanol in the container is maintained at different temperatures using a heater. Figure 1 depicts the schematic diagram of the experimental setup used in the present study. It consists of three main components, namely, (i) an acrylic container with an aluminum metallic base placed on a heater, (ii) a droplet generation and dispensing system, and (iii) a high-speed imaging and illumination system. The side walls of the container are made up of an acrylic sheet (of a thickness $4 \mathrm{~mm}$ ) in order to provide optical access to the high-speed imaging system. Due to the high heat conductivity, an aluminum plate (of a thickness of $12 \mathrm{~mm}$ ) is used as the base of the container, which helps to maintain the ethanol in the container at various elevated temperatures. A sufficiently large container size is used so that the reflected waves from the walls have negligible effects on the coalescence dynamics.

The temperature of the heater is varied, and six values of the temperature of the ethanol-air interface in the pool $\left(T_{s}\right)$, namely, $25^{\circ} \mathrm{C}, 30^{\circ} \mathrm{C}, 35^{\circ} \mathrm{C}, 40^{\circ} \mathrm{C}, 45^{\circ} \mathrm{C}$, and $50^{\circ} \mathrm{C}$, are considered. Figure 2 shows the temperature variation inside the liquid pool. Here, $y=0$ and $y=15 \mathrm{~mm}$ represent the top of the aluminum plate placed on the heater and the ethanol-air interface of the ethanol pool, respectively. The heater temperature is fixed at a slightly higher value, $T_{s}+3{ }^{\circ} \mathrm{C}$, to keep the ethanol-air interface temperature at a particular value of $T_{s}$. The ethanol inside the container placed in the heater is stirred during the heating process to obtain the desired homogeneous temperature. After the desired interface temperature is reached, we stop stirring and measure the temperatures inside the ethanol pool at different heights (namely, $y=0 \mathrm{~mm}, 4 \mathrm{~mm}, 8 \mathrm{~mm}, 12 \mathrm{~mm}$, and $15 \mathrm{~mm}$ ) using the K-type thermocouples. Figure 2 presents the temperature variations in the vertical direction for different values of $T_{s}$. It is observed that temperature measurement uncertainty is about $\pm 1.5^{\circ} \mathrm{C}$ that is associated with the thermocouple calibration error. The fluid properties of ethanol at different temperatures are listed in Table I.

The droplet generation and dispensing system consists of a syringe pump (SP-810) fitted with different grades of blunt edge needles, namely, $12 \mathrm{G}, 14 \mathrm{G}, 16 \mathrm{G}, 18 \mathrm{G}$, and $20 \mathrm{G}$. This system is also
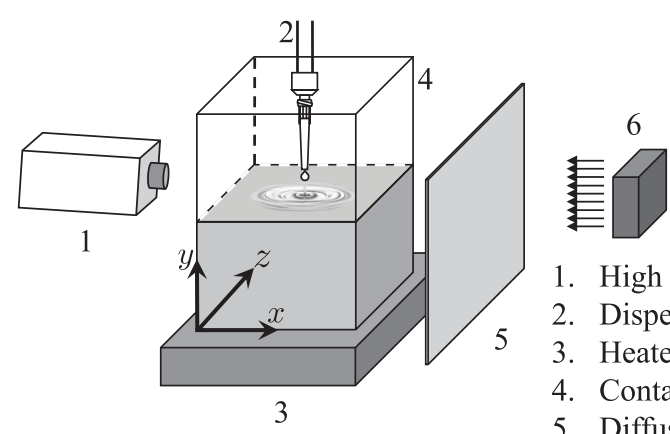

1. High speed camera

2. Dispensing needle

3. Heater

4. Container

5. Diffuser sheet

6. Light source

FIG. 1. Schematic of the experimental setup. It consists of a high-speed camera, a dispensing needle, a heater, an acrylic container with an aluminum metallic base, a diffuser sheet, and a light source. The inner dimension of the container is $48 \times 48 \times 48 \mathrm{~mm}^{3}$. The thickness of the acrylic side walls of the container is $4 \mathrm{~mm}$, and the thickness of the aluminum metallic base is $12 \mathrm{~mm}$. The height of ethanol in the container is $15 \mathrm{~mm}$.

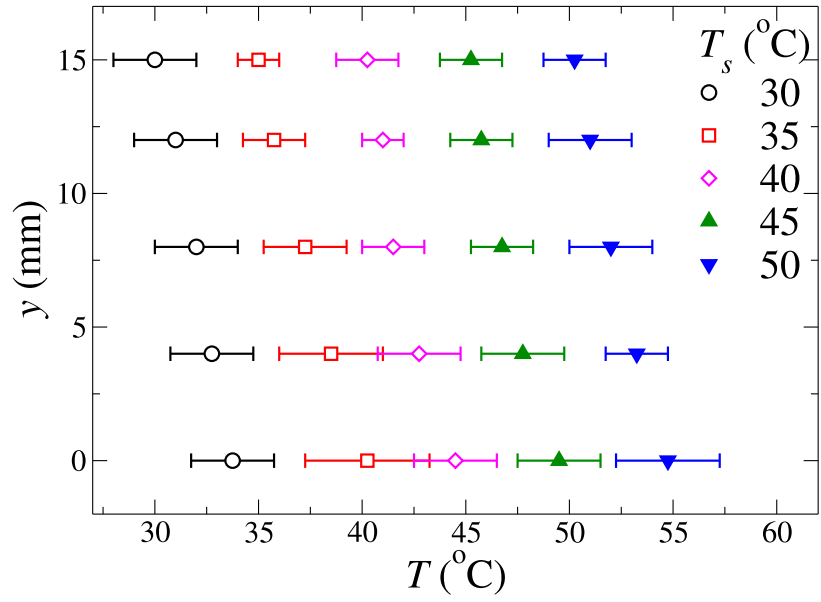

FIG. 2. The temperature profiles in the ethanol pool for different values of ethanolair interface temperature, $T_{s}$.

facilitated by means of a traversing mechanism to anchor the needle height from the ethanol-air interface of the pool. By changing the ethanol volumetric flow rate and using different needles, we create different sizes of ethanol droplets at $25^{\circ} \mathrm{C}$. The drops released from the needles come into contact with the ethanol pool kept at various temperatures. In our experiments, at the time of release, the distance between the bottom of the drops and the free-surface of the ethanol pool $(h)$ is kept constant at $0.4 \pm 0.05 \mathrm{~mm}$, except when we examine the effect of the height the dispensing needle tip from the free-surface $(H)$ on the coalescence dynamics.

A high-speed imaging and lighting system (shadowgraphy) consists of a Photron camera (model: Fastcam SA1.1) along with a Nikon lens of $50 \mathrm{~mm}$ focal length is used to record the coalescence dynamics. The camera is kept at an angle of $7^{\circ}$ to the horizontal, as shown in Fig. 1, to visualize the droplet coalescence at the horizontal ethanol-air interface. A diffused backlit Light Emitting Diode (LED) illumination system (model $900445 \mathrm{~lm}, 12000 \mathrm{~lm}$, Visual Instrumentation Corporation) is used to illuminate the test section of the transparent wall container. Images at a resolution of $320 \times 240$ pixels are recorded at a frame rate of $10000 \mathrm{fps}$ with an exposure time of $10 \mu \mathrm{s}$. The recorded images are analyzed using the Matlab software. For each value of $T_{s}$, experiments are repeated five times to

TABLE I. The values of surface tension $(\sigma)$, dynamic viscosity $\left(\mu_{l}\right)$, and density $\left(\rho_{l}\right)$ of ethanol at different temperatures. ${ }^{34-36}$

\begin{tabular}{lccc}
\hline \hline Temperature $\left({ }^{\circ} \mathrm{C}\right)$ & $\sigma(\mathrm{mN} / \mathrm{m})$ & $\mu_{l}(\mathrm{mPa} \mathrm{s})$ & $\rho_{l}\left(\mathrm{~kg} / \mathrm{m}^{3}\right)$ \\
\hline 25 & 21.93 & 1.20 & 783.92 \\
30 & 21.48 & 1.01 & 778.91 \\
35 & 21.04 & 0.87 & 773.85 \\
40 & 20.64 & 0.87 & 768.74 \\
45 & 20.22 & 0.73 & 763.57 \\
50 & 19.83 & 0.71 & 758.34 \\
\hline \hline
\end{tabular}


TABLE II. Comparison of the thermal diffusion time scale $\left(t_{c}=D_{p}{ }^{2} / \alpha\right)$ and residence time $\left(t_{r}\right)$ for different sizes of ethanol drop. The thermal diffusivity $(\alpha)$ of ethanol is $0.0827 \mathrm{~mm}^{2} / \mathrm{s}$

\begin{tabular}{lll}
\hline \hline$D_{p}(\mathrm{~mm})$ & $t_{c}(\mathrm{~s})$ & $t_{r}(\mathrm{~s})$ \\
\hline 1.71 & 35.3 & 0.063 \\
1.83 & 40.5 & 0.043 \\
2.05 & 50.8 & 0.030 \\
2.10 & 53.3 & 0.025 \\
\hline
\end{tabular}

ensure repeatability. The reported uncertainty is estimated based on the standard deviation obtained from the five repetitions.

A droplet of ethanol placed slowly on the free-surface of the ethanol pool floats on the free-surface for some time (residence time, $\left.t_{r}\right)$, which depends on pool temperature $\left(T_{s}\right)$ and the impact height $(H)$ and the size of the primary droplet $\left(D_{p}\right)$, before draining inside the ethanol pool. The comparison of the residence time and the thermal diffusion time scale is provided in Table II at $T_{s}=25^{\circ} \mathrm{C}$ (isothermal condition). It can be seen that, in our experiment, the residence time is much shorter than the time scale associated with thermal diffusion. In all our experiments, it is observed that the coalescence phenomenon occurs very quickly; the time taken for this process is always less than $75 \mathrm{~ms}$. Thus, a freely falling drop does not get enough time to develop the Marangoni flow. This point has also been discussed in the introduction section. At later times, we also observe a partial coalescence behavior for some set of parameters. In Sec. III, the coalescence dynamics of an ethanol drop observed for different sets of parameters is analyzed.

\section{RESULTS AND DISCUSSION}

The primary objective of this study is to investigate the coalescence dynamics of an ethanol drop that is slowly placed on an ethanol-air interface in an ethanol pool maintained at different elevated temperatures $\left(T_{s}\right)$. We also carried out a parametric study by varying the diameter of the primary drop $\left(D_{p}\right)$ released from a dispensing needle and the height of the needle tip from the ethanol-air interface $(H)$ at different values of $T_{s}$.

We begin the presentation of our results by analyzing the temporal evolutions of the coalescence dynamics of an ethanol drop of $D_{p}=1.83 \mathrm{~mm}$ at $T_{s}=25^{\circ} \mathrm{C}, 35^{\circ} \mathrm{C}$, and $45^{\circ} \mathrm{C}$ in Fig. 3 (Multimedia view). This figure demonstrates the topological changes that the drop exhibits during the coalescence process when it is gently placed on the ethanol-air interface of the ethanol pool. The impact velocity $(U)$ of the drop that is calculated using two consecutive frames of the drop just before it touches the interface is found to be very small $(\approx 0.03 \mathrm{~m} / \mathrm{s})$. Note that, in order to calculate the terminal velocity, the coordinates of the center of gravity of the drop in different instants are obtained, and then the velocity $(U)$ of the drop is calculated as $U=\Delta y / \Delta t$. Here, $\Delta y=y_{n}-y_{n-m}$, wherein $y_{n}$ is the $y$-coordinate of the drop's center of gravity just before the it touches the free surface (say at frame " $n$ ") and $y_{n-m}$ is the location of the drop's center of gravity at $n-m$ frame; $\Delta t$ represents the time difference between these frames. We use $m=20$, which corresponds to $\Delta t=0.002 \mathrm{~s}$ as the acquisition of the images is performed at $10000 \mathrm{fps}$, and $\Delta y$ is the distance travel by the drop in $\Delta t$. Figure 3(a) (Multimedia view) demonstrates the coalescence dynamics of an ethanol drop in an isothermal condition, i.e., when the drop and ethanol pool are at the same temperature $\left(25^{\circ} \mathrm{C}\right)$. Figures $3(\mathrm{~b})$ and $3(\mathrm{c})$ show the coalescence dynamics of an ethanol drop in non-isothermal conditions,
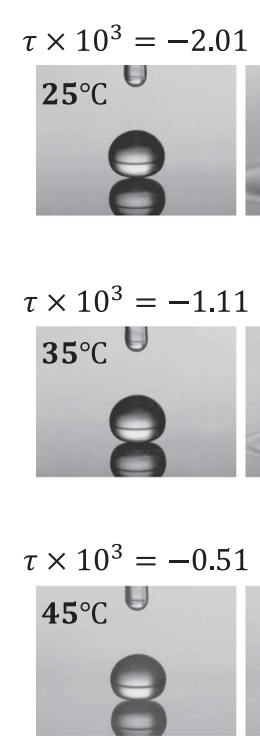

0.00

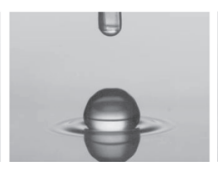

0.42

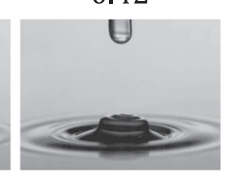

0.42

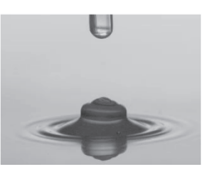

(a)
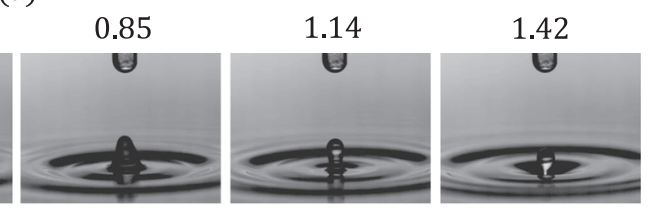

(b)
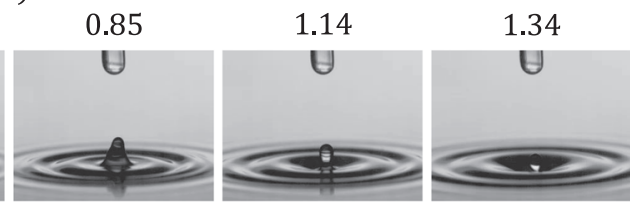

(c)
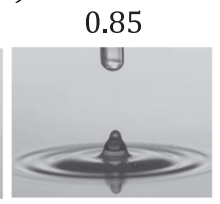

1.14
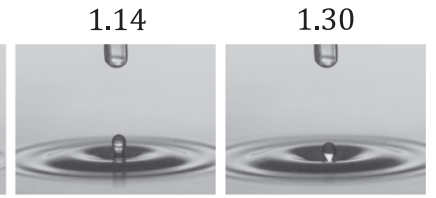

FIG. 3. The temporal evolutions of the coalescence dynamics of an ethanol drop of diameter $D_{p}=1.83 \mathrm{~mm}$ for different values of ethanol-air interface temperature in the pool, $T_{s}$. The dimensionless time, $\tau=t / t_{c}$, is written at the top of each image, such that $\tau=0$ represents the instant when the drop starts draining in the liquid pool. The dynamics at $T_{s}=25^{\circ} \mathrm{C}$ [panel (a)], $T_{s}=45^{\circ} \mathrm{C}$ [panel (b)], and $T_{s}=55^{\circ} \mathrm{C}$ [panel (c)] are demonstrated, respectively. Multimedia views: https://doi.org/10.1063/5.0007402.1; https://doi.org/10.1063/5.0007402.2; and https://doi.org/10.1063/5.0007402.3 
wherein the drop is at $25^{\circ} \mathrm{C}$ and the ethanol pool is at $35^{\circ} \mathrm{C}$ and $45^{\circ} \mathrm{C}$, respectively (see Figs. 3(b) and 3(c) (Multimedia view)]. The first column in panels (a)-(c) is associated with the instant at which the drop just touches the ethanol-air interface of the pool. The second column corresponds to the onset of the coalescence/draining of the drop inside the pool. The number written at the top of each image represents the dimensionless time, $\tau=t / t_{c}$. The time, $t$, is calculated in such a way that dimensionless time, $\tau$ is zero at the onset of the coalescence, and the negative and positive values of $\tau$ represent the periods before and after the onset of coalescence, respectively. The temporal evolution of the drop dynamics is presented in the subsequent columns in Fig. 3 (Multimedia view). The first pinchoff of the daughter droplet (partial coalescence dynamics) is shown in the last panel for each value of $T_{s}$.

In the isothermal condition $\left(T_{s}=25^{\circ} \mathrm{C}\right)$, it can be seen in Fig. 3(a) (Multimedia view) that the dimensionless residence time of the drop is 2.01. During this period, the drop floats on the ethanolair interface in the pool until the air trapped between the drop and the free-surface is drained out. At $\tau=0$, the drop starts to drain inside the pool, and a neck is formed at the contact point of the drop and the free-surface, which expands rapidly due to high capillary pressure near the contact region (at $\tau=0.42$; third column). Due to this, upward moving capillary waves are generated, thereby forming a liquid column (at $\tau=0.85$; fourth column). This is followed by a necking process near the contact region (at $\tau=1.14$; fifth column). Subsequently, the diameter of the neck is reduced due to the inward pull of the surface tension, and a daughter droplet is detached (at $\tau$ $=1.42$; sixth column). This pinch-off of the daughter droplet occurs when the vertical collapse rate decreases due to the upward pull exerted by the capillary waves, and thereby the horizontal collapse succeeds in merging the neck and producing a daughter droplet. The coalescence dynamics of a drop in the isothermal condition has been studied by several researchers (see, for instance, Refs. 11, 13, 14, 16, 17,37 , and 38 as discussed in the introduction section).

A qualitatively similar coalescence dynamics is also observed in the non-isothermal cases presented in Figs. 3(b) and 3(c) (Multimedia view) for $T_{s}=35^{\circ} \mathrm{C}$ and $45^{\circ} \mathrm{C}$, respectively. However, inspection of the dimensionless residence time and pinch-off time (defined as the difference between the instants at which the first pinch-off occurs and the onset of coalescence) decreases as the temperature of the ethanol pool increases. The dimensionless residence times of the drop for $T_{s}=35^{\circ} \mathrm{C}$ and $45^{\circ} \mathrm{C}$ are 1.11 and 0.51 , respectively. It is to be noted that increasing the temperature decreases the surface tension, viscosity, and density of ethanol. ${ }^{34-36}$ As the ethanol drop at $25^{\circ} \mathrm{C}$ comes in contact with a hotter ethanol free-surface in the pool, the Marangoni convection starts to develop due to the gradient of surface tension resulting from the temperature gradient along the ethanol-air interface. Increasing the temperature of the ethanol pool increases this Marangoni convection. However, unlike Geri et al., ${ }^{19}$ in our case, due to the low residence time, the drop did not get sufficient time to develop the fully developed Marangoni convection. The mechanism of the coalescence dynamics observed in our non-isothermal cases is discussed next.

In Fig. 4, we analyzed the forces acting on the drop when it floats at the free-surface of the ethanol pool to explain the decrease in the residence time with the increase in pool temperature. The weight of the drop creates a depression on the free-surface with an air film trapped in between the drop and ethanol-air interface in

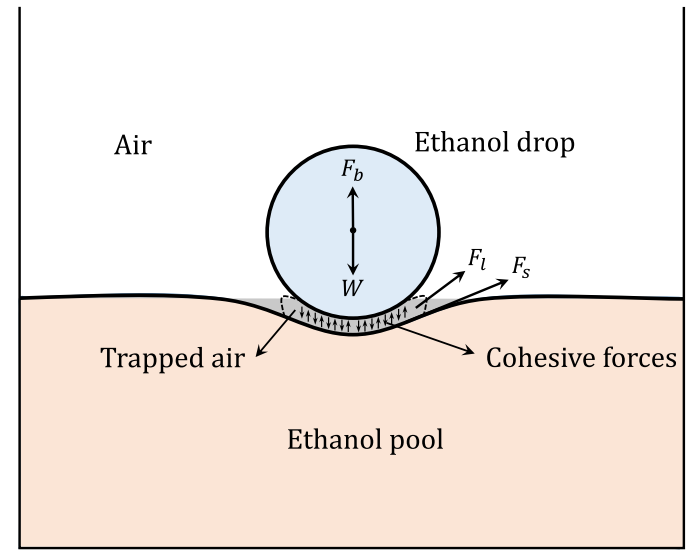

FIG. 4. A sketch of different forces acting on a drop that is gently placed on the free-surface of a liquid pool. The various forces experienced by the drop are the weight of the drop $(W)$, buoyancy force $\left(F_{b}\right)$, surface tension force acting along the curved free-surface in the pool $\left(F_{l}\right)$, and lift force due to the squeezed air $\left(F_{s}\right)$.

the pool. The various forces experienced by the drop during this time are the weight of the drop acting in the downward direction $(W)$, upward buoyancy force $\left(F_{b}\right)$, surface tension force acting along the curved free-surface in the pool $\left(F_{l}=2 \pi r_{c} \sigma\right)$, and lift force due to the squeezed air which drains outward $\left(F_{s}=\rho_{a} v^{2} A / 2\right)$. Here, $\rho_{a}$ is the density of air, $v$ is the velocity of squeezed out air, and $A$ and $r_{c}$ are the surface area and the radius of the curved surface, respectively. When the drop floats on the free-surface of the pool, the normal components of these forces acting on the drop balance each other. The surface tension delays coalescence by making the free-surface curve to accommodate the drop. Increasing temperature decreases the surface tension, which in turn decreases the free-surface depression. This is evident in Fig. 3 (Multimedia view) (second column), where it can be seen that, at the onset of coalescence $(\tau=0)$, the radius of the curved free-surface in the isothermal case $\left(T_{s}=25^{\circ} \mathrm{C}\right)$ is significantly large (almost the same as the radius of the drop). It can be seen that the free-surface depression decreases with increasing the temperature of ethanol in the pool. At $T_{s}=45^{\circ} \mathrm{C}$, the free-surface is almost flat at the onset of the coalescence. The decrease in the free-surface depression results in faster coalescence, i.e., decreases the residence time, $t_{r}$, of the drop. At the later stage $(\tau \geq 0)$, the drop touches the free-surface after draining the air cushion. The Marangoni convection from hotter to colder regions (i.e., in the upward direction) builds up once the drop touched the ethanol-air interface in the pool. Increasing the temperature gradient between the drop and ethanol pool increases the surface tension gradient, which in turn enhances the upward moving capillary waves due to Marangoni convection. As a consequence, increasing temperature reduces the rate of vertical collapse, resulting in faster daughter droplet formation [see Fig. 3 (Multimedia view)] due to the more dominant horizontal collapse.

To gain more insight, in Fig. 5, we compare the temporal evolutions of drop profiles during the coalescence process at $T_{s}=25^{\circ} \mathrm{C}$ (isothermal case) and $45^{\circ} \mathrm{C}$ (non-isothermal case). In both cases, the drop diameter, $D_{p}$, is kept constant at $1.71 \mathrm{~mm}$. It can be seen that 


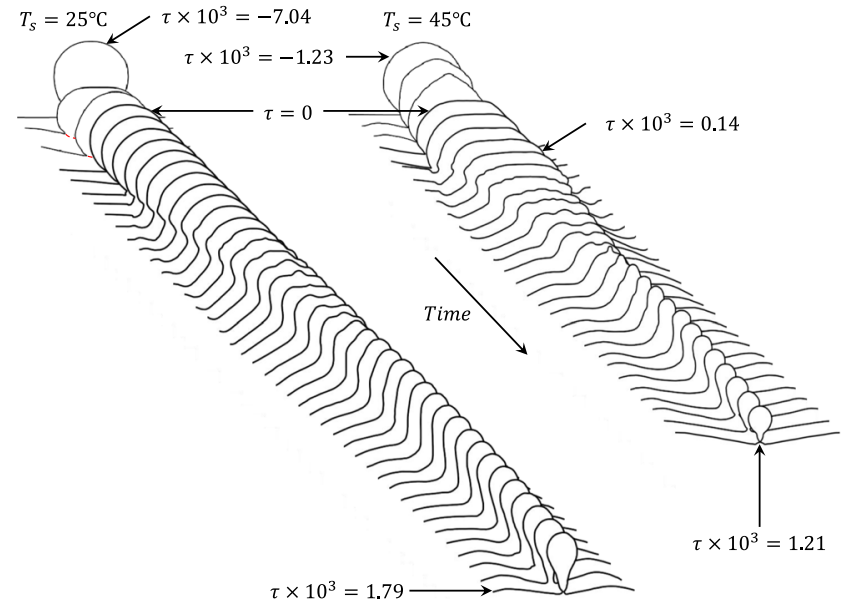

FIG. 5. Temporal evolutions of drop profile at $T_{s}=25^{\circ} \mathrm{C}$ and $45^{\circ} \mathrm{C}$. In both the cases, the diameter of the primary drop, $D_{p}=1.71 \mathrm{~mm}$. The value of $\Delta \tau$ between two consecutive profiles after the drop touches the free-surface in the pool is 0.046 $\times 10^{-3}$.

the drop is almost spherical in both cases when it is sufficiently away from the free-surface of the pool. Note that as the drop approaches the free-surface, it becomes difficult to obtain the distinct interfaces of the drop and free-surface from the image processing. It can be seen that for $\tau<0$, the drop undergoes vertical oscillations, which is more in the isothermal case as compared to the non-isothermal case. These vertical oscillations in the drop try to regain the air cushion between the drop and the free-surface in the pool before the coalescence starts at $\tau=0$. It can be seen in both cases that as the drop starts to drain inside the pool, the neck between the drop and the free-surface expands rapidly due to high capillary pressure near the contact region. In the isothermal case, due to the resultant upward moving capillary waves, a vertical liquid column is created. As it grows, the horizontal collapse due to the inward pull of the surface tension shrinks the neck region and a daughter droplet is created at $\tau$ $=1.79 \times 10^{-3}$. In Fig. 5 , it can be seen at $\tau=1.79 \times 10^{-3}$ and $\tau=1.21$ $\times 10^{-3}$ for $T_{s}=25^{\circ} \mathrm{C}$ and $45^{\circ} \mathrm{C}$ that the interface of the drop and the pool form a sharp cone angle, after which blunting of this cone angle and capillary waves creeping on the droplet surface are observed. This is the instant at which a daughter droplet is formed. The coalescence time is defined as the difference between the moment the capillary waves begin to ascend on the droplet surface due to the effect of Marangoni and the detachment of the daughter droplet. In contrast to the isothermal case, for $T_{s}=45^{\circ} \mathrm{C}$, the drop spreads more on the free-surface in the pool during the draining stage. A significantly flat drop summit is observed at $T_{s}=45^{\circ} \mathrm{C}$. In this case, the intensity of the upward moving capillary waves produced due to the expansion of the neck region between the drop and the freesurface are enhanced by the resultant Marangoni convection due to the temperature gradient. This is evident after $\tau=0.14 \times 10^{-3}$ for $T_{s}=45^{\circ} \mathrm{C}$. This in turn prevents the column to drain into the pool, and the neck region becomes thinner and detached from the freesurface of the pool due to the horizontal collapse at $\tau=1.21 \times 10^{-3}$. Thus, the temperature gradient promotes the partial coalescence phenomenon.

Next, we investigate the effect of the height of the needle tip from the free-surface of the pool $(H)$ on the coalescence dynamics of a drop of $D_{p}=1.71 \mathrm{~mm}$, as shown in Fig. 6 (Multimedia view). Figures 6(a) and 6(b) (Multimedia view) present the dynamics for $H=3.66 \mathrm{~mm}$ and $H=7.83 \mathrm{~mm}$, respectively. The dynamics for $H=3.66 \mathrm{~mm}$ and $H=7.83 \mathrm{~mm}$ are also presented as Figs. 6(a) and 6(b) (Multimedia view), respectively. The first and second columns in each panel represent the frames when the drop touches the free-surface and starts to coalesce in the pool, respectively. The columns that follow demonstrate the dynamics as the time progresses. Increasing $H$ increases the potential energy of the drop, which is converted into kinetic energy, thereby increasing the impact velocity of the drop falling into the ethanol pool.

\section{(a) Partial coalescence}

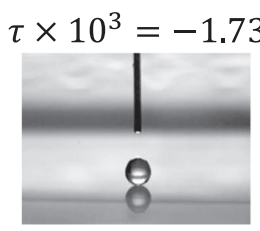

0.00
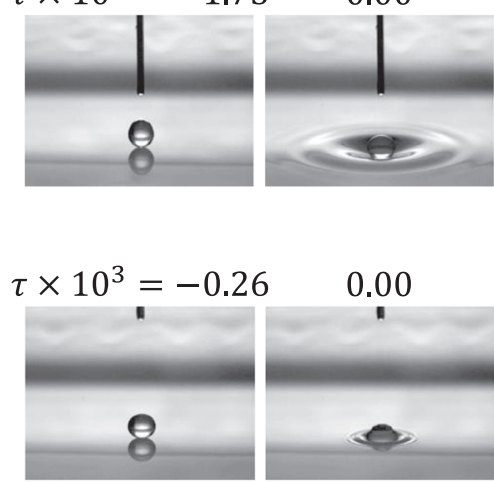

0.00

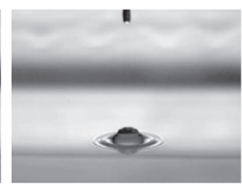

0.23
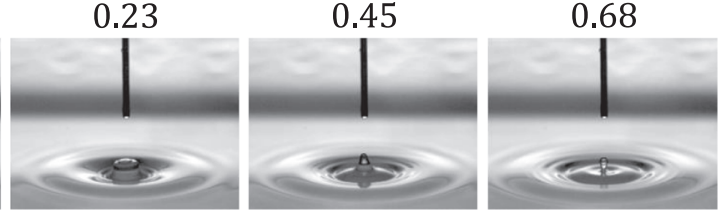

0.68
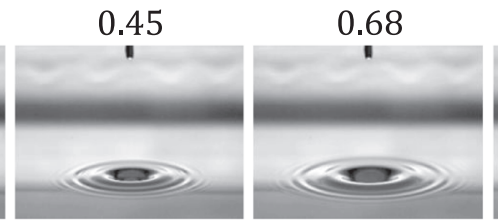

0.79

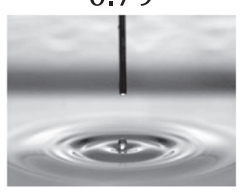

0.79

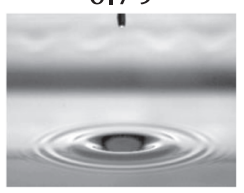

FIG. 6. The effect of the height of the needle tip from the free-surface of the pool, $H$, on the coalescence dynamics of an ethanol drop of primary diameter, $D_{p}=1.71 \mathrm{~mm}$ at $T_{s}=30^{\circ} \mathrm{C}$. (a) $H=3.66 \mathrm{~mm}$ and (b) $H=7.83 \mathrm{~mm}$. The value of the dimensionless time, $\tau$, is shown at the top of each panel. $\tau=0$ represents the instant at the onset of coalescence. The dynamics at $H=3.66 \mathrm{~mm}$ [panel (a)] and $H=7.83 \mathrm{~mm}$ [panel (b)] are demonstrated, respectively. Multimedia views: https://doi.org/10.1063/5.0007402.4; https://doi.org/10.1063/5.0007402.5 
The value of the impact velocities of the drop for $H=3.66 \mathrm{~mm}$ and $H=7.83 \mathrm{~mm}$ are $0.12 \mathrm{~m} / \mathrm{s}$ and $0.32 \mathrm{~m} / \mathrm{s}$, respectively. It can be seen that the drop floats on the free-surface for a longer time $(\tau=1.73)$ for $H=3.66 \mathrm{~mm}$, as compared to $H=7.83 \mathrm{~mm}$. The drop also demonstrates the partial coalescence phenomenon at $\tau=0.79$ for $H=3.66 \mathrm{~mm}$, whereas this behavior does not occur for $H=7.83 \mathrm{~mm}$, and the drop completely coalesce inside the pool creating a much bigger depression on the free-surface at the same dimensionless time. Close inspection of Figs. 6(a) and 6(b) (Multimedia view) also reveals that increasing the impact velocity decreases with the width of the crater developed at the interface. It indicates that, in the case of a drop with a high impact velocity, the upward force to support the drop becomes insignificant as compared to a gently placed drop with a negligible impact velocity. Increasing the impact velocity of the drop significantly decreases the vertical component of the surface tension force and also decreases the buoyant force as the swept volume is displaced farther away. Complete coalescence is observed in the case of a high impact velocity drop. Thus, as expected, it can be inferred that, in addition to the fluid properties, the needle height also plays an important role in the coalescence dynamics.

In Fig. 7, we present the regimes associated with the partial coalescence (black circles) and complete coalescence without the formation of a daughter droplet (red squares) in the $T_{s}-H$ space for a primary drop of size, $D_{p}=1.71 \mathrm{~mm}$. To ensure repeatability, experiments are performed five times for each set of values of $T_{s}$ and $H$. It can be seen that, in the isothermal condition $\left(T_{s}=25^{\circ} \mathrm{C}\right)$, the partial coalescence phenomenon is observed for all $H$ values considered in the present study. For $T_{s} \geq 30{ }^{\circ} \mathrm{C}$, the partial coalescence phenomenon is only observed for low values of $H$. This is due to the competition between the upward moving capillary waves and the impact velocity of the drop. In other words, increasing the momentum force generated by a falling droplet (i.e., increasing the height of the nozzle) decreases the intensity of the upward moving capillary waves, which allows the drop to penetrate into the pool leading to complete coalescence. It can be seen that the critical value of $H$ at which the drop changes its behavior from partial coalescence to

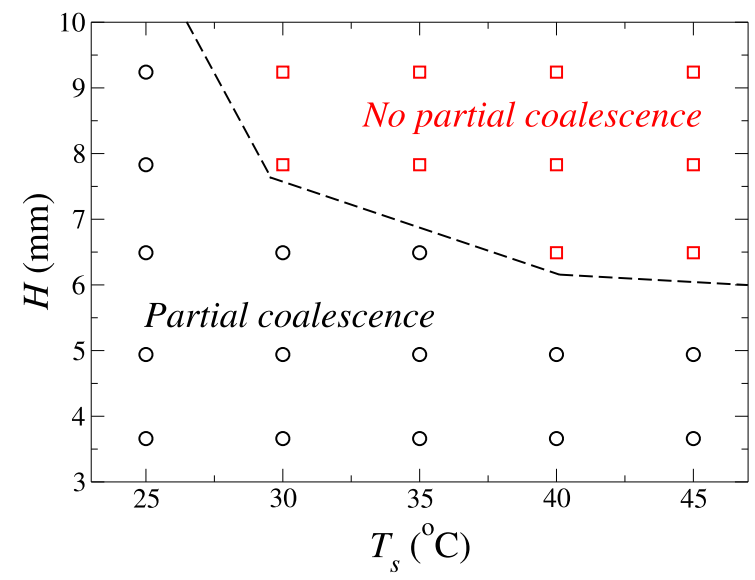

FIG. 7. Regime map showing the partial coalescence and complete coalescence without forming a daughter droplet in the $T_{s}-H$ space. Here, the diameter of the primary drop, $D_{p}=1.71$.

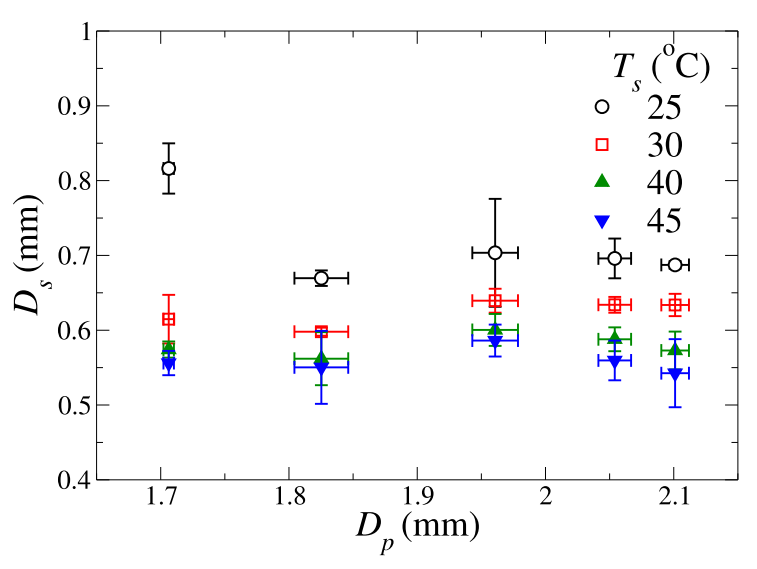

FIG. 8. Variations in the diameter of the first daughter droplet $\left(D_{s}\right.$ in $\left.\mathrm{mm}\right)$ with the diameter of the primary drop $\left(D_{p}\right.$ in $\left.\mathrm{mm}\right)$ for different values of $T_{s}$.

complete coalescence decreases with an increase in the temperature of the ethanol pool.

Then, we conduct a parametric study by varying the diameter of the primary drop injected from the needle $\left(D_{p}\right)$ and ethanol pool temperature, $T_{s}$. The height of the bottom of the drop from the free-surface is fixed at $h=0.4 \pm 0.05 \mathrm{~mm}$. The parameters are chosen in such a way that the drop exhibits partial coalescence for all values of $T_{s}$. In Fig. 8, we present the variations in the diameter of the daughter droplet $\left(D_{s}\right)$ obtained for different sizes of the primary drop $\left(D_{p}\right)$ at different values of $T_{s}$. Five primary drop sizes (i.e., $D_{p}=1.71 \mathrm{~mm}, 1.83 \mathrm{~mm}, 1.96 \mathrm{~mm}, 2.05 \mathrm{~mm}$, and $2.10 \mathrm{~mm}$ ) and four values of $T_{s}$ (i.e., $T_{s}=25^{\circ} \mathrm{C}, 30^{\circ} \mathrm{C}, 40^{\circ} \mathrm{C}$, and $45^{\circ} \mathrm{C}$ ) have been considered to generate this plot. It can be seen that increasing temperature decreases the size of the daughter droplet for all sizes of the primary drop considered. This is because, as discussed above, increasing temperature promotes the drainage of the primary drop, thereby leaving a smaller volume of ethanol in the column for the daughter droplet creation. It can be observed that increasing $D_{p}$

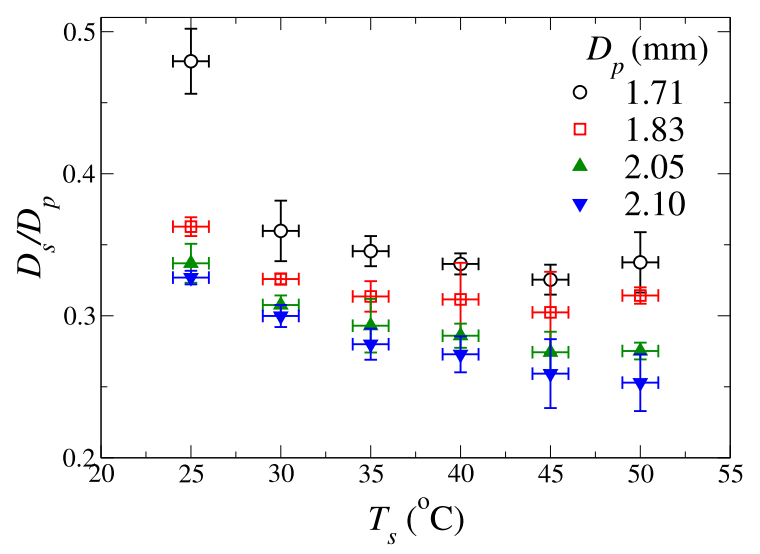

FIG. 9. The variations of $D_{s} / D_{p}$ with $T_{s}$ for different values of primary diameter of the ethanol drop, $D_{p}$. 


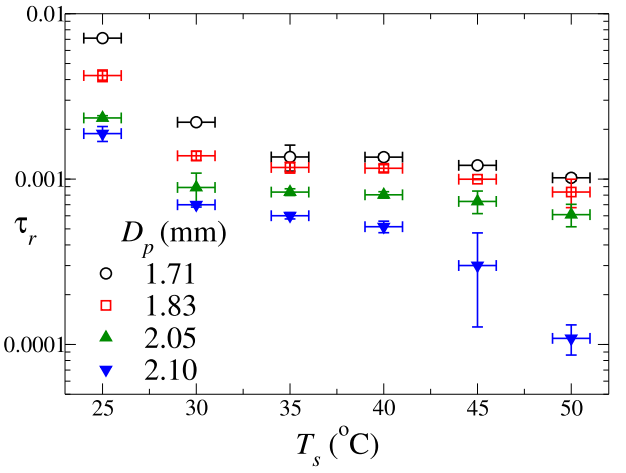

(a)

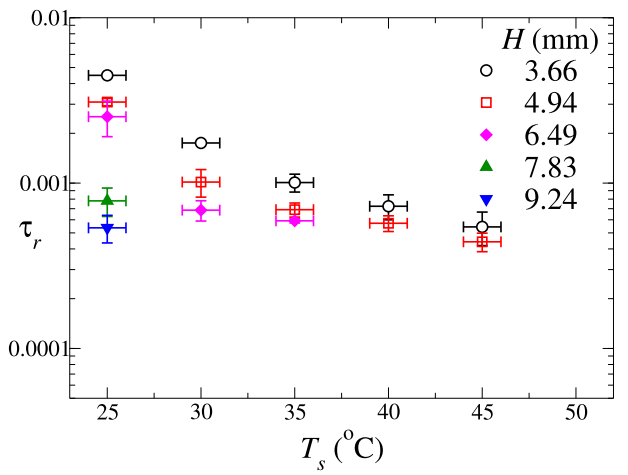

(b)
FIG. 10. Variations of dimensionless residence time, $\tau_{r}=t_{r} / t_{c}$, with the temperature at the free-surface the ethanol pool, $T_{s}$, for different values of (a) the diameter of the primary drop, $D_{p}$, when the tip of the drop is at $0.4 \pm 0.05 \mathrm{~mm}$ from the free-surface, and (b) height of the needle tip from the ethanol-air interface, $H$, for $D_{p}=1.83 \mathrm{~mm}$. decreases the size of the daughter droplet to an asymptotic value. Inspection of this figure also reveals that $D_{s} / D_{p}$ is about 0.5 for $D_{p}=1.71 \mathrm{~mm}$ in the isothermal condition, which is similar to the value observed in the previous studies. ${ }^{14,17}$

Figure 9 shows the variations in $D_{s} / D_{p}$ with $T_{s}$ for different values of $D_{p}$. It can be seen that increasing the pool temperature, $T_{s}$, decreased the value of $D_{s} / D_{p}$ for all sizes of the primary drop. In all the cases, the value of $D_{s} / D_{p}$ is less than 0.5 . Close inspection of Figs. 8 and 9 also reveals that increasing the size of the primary drop decreases the values of $D_{s} / D_{p}$ for all values of $T_{s}$ considered. In an isothermal system, Chen et al. ${ }^{14}$ also showed that the drop goes from the inertio-capillary regime to the gravity regime, thereby decreasing the value of $D_{s} / D_{p}$ as we increase the size of the primary drop.

Finally, the variations of the dimensionless residence time, $\tau_{r}$, with the free-surface temperature of the pool, $T_{s}$, are presented for different values of $D_{p}$ and $H$ in Figs. 10(a) and 10(b), respectively. It can be seen in Fig. 10(a) that increasing the temperature of the ethanol pool decreases the residence time for all values of $D_{p}$. Increasing $D_{p}$ increased the weight of the drop, which promotes the draining of the trapped air in between the drop and the free-surface of the pool. Increasing the temperature of the pool decreases the surface tension, and thus the free-surface could not become curved to hold the drop and coalescence starts. Thus, increasing temperature decreases the residence time of the drop [see Fig. 10(b)]. It can also be seen in Fig. 10(b) that increasing height increases the impact velocity of the drop, which drains the trapped air easily and reduces the residence time.

\section{CONCLUDING REMARKS}

In this study, we revisit the coalescence dynamics of a drop (at $25^{\circ} \mathrm{C}$ ) slowly placed on the free-surface of a pool maintained at different temperatures $\left(T_{s}\right)$ by conducting experiments. The experimental setup consists of a high-speed camera, a dispensing needle, a heater, an acrylic container of inner dimension $48 \times 48 \times 48 \mathrm{~mm}^{3}$ with an aluminum metallic base, a diffuser sheet, and a light source. The container is filled with ethanol to a height of up to $15 \mathrm{~mm}$. It is observed that the ethanol drop exhibits a residence phase, when it floats on the free-surface before the coalescence begins. Subsequently, the complete coalescence and partial coalescence dynamics are observed for different sets of parameters considered. In our experiments, as we considered the coalescence of a freely falling drop, the residence time is much shorter than the time scale associated with thermal diffusion. Thus, the drop does not get enough time to develop the Marangoni flow during the residence phase, which was found to play a key role in delaying the coalescence in the previous studies.

We carried out a parametric study by varying the diameter of the primary drop $\left(D_{p}\right)$ released from a dispensing needle and the height of the needle tip from the ethanol-air interface $(H)$ at different values of $T_{s}$. It is found that increasing the temperature of the ethanol pool reduces the residence time of the drop. This phenomenon is explained by analyzing the forces acting on the drop and the capillary waves generated due to the temperature gradient between the drop and the ethanol pool. As expected, due to the gravity effect, increasing the size of the primary drop also decreases the residence time. The partial coalescence phenomenon is observed for low impact height, and it is found that the diameter of the daughter droplet decreases as the size of the primary drop and pool temperature are increased. A regime map designating the complete coalescence and partial coalescence dynamics is plotted in the $T_{s}-H$ space.

\section{ACKNOWLEDGMENTS}

K.C.S. and P.K. thank the Science and Engineering Research Board, India, for their financial support (Grant Nos. MTR/2017/ 000029 and ECR/2015/000365).

\section{DATA AVAILABILITY}

The data that support the findings of this study are available from the corresponding author upon reasonable request.

\section{REFERENCES}

${ }^{1}$ H. A. Stone, A. D. Stroock, and A. Ajdari, "Engineering flows in small devices," Annu. Rev. Fluid Mech. 36, 381-411 (2004).

${ }^{2}$ S. T. Thoroddsen, T. G. Etoh, and K. Takehara, "High-speed imaging of drops and bubbles," Annu. Rev. Fluid Mech. 40, 257-285 (2008).

${ }^{3}$ F. H. Zhang, M.-J. Thoraval, S. T. Thoroddsen, and P. Taborek, "Partial coalescence from bubbles to drops,” J. Fluid Mech. 782, 209-239 (2015). 
${ }^{4} \mathrm{~K}$. Haldar and S. Chakraborty, "Investigation of chemical reaction during sodium alginate drop impact on calcium chloride film," Phys. Fluids 31(7), 072102 (2019). ${ }^{5}$ M. Kumar, R. Bhardwaj, and K. C. Sahu, "Coalescence dynamics of a droplet on a sessile droplet," Phys. Fluids 32(1), 012104 (2020).

${ }^{6}$ R. Abdelaziz, D. Disci-Zayed, M. K. Hedayati, J. H. Pöhls, A. U. Zillohu, B. Erkartal, V. S. K. Chakravadhanula, V. Duppel, L. Kienle, and M. Elbahri, "Green chemistry and nanofabrication in a levitated leidenfrost drop," Nat. Commun. $\mathbf{4}$, 2400 (2013).

${ }^{7} \mathrm{~B}$. Schwenzer, "Leidenfrost drops prove to be versatile nanoreactors," MRS Bull. 39(1), 7 (2014).

${ }^{8} \mathrm{M}$. Mrinal, X. Wang, and C. Luo, "Self-rotation-induced propulsion of a leidenfrost drop on a ratchet," Langmuir 33(25), 6307-6313 (2017).

${ }^{9}$ E. Mogilevskiy, "Levitation of a nonboiling droplet over hot liquid bath," Phys. Fluids 32(1), 012114 (2020).

${ }^{10}$ B. Ray, G. Biswas, and A. Sharma, "Regimes during liquid drop impact on a liquid pool," J. Fluid Mech. 768, 492-523 (2015).

${ }^{11}$ G. E. Charles and S. G. Mason, "The mechanism of partial coalescence of liquid drops at liquid/liquid interfaces," J. Colloid Sci. 15(2), 105-122 (1960).

${ }^{12} \mathrm{~S}$. T. Thoroddsen and K. Takehara, "The coalescence cascade of a drop," Phys. Fluids 12(6), 1265-1267 (2000).

${ }^{13}$ H. Aryafar and H. P. Kavehpour, "Drop coalescence through planar surfaces," Phys. Fluids 18(7), 072105 (2006).

${ }^{14} \mathrm{X}$. Chen, S. Mandre, and J. J. Feng, "Partial coalescence between a drop and a liquid-liquid interface," Phys. Fluids 18(5), 051705 (2006).

${ }^{15} \mathrm{X}$. Chen, S. Mandre, and J. J. Feng, "An experimental study of the coalescence between a drop and an interface in Newtonian and polymeric liquids," Phys. Fluids 18, 092103 (2006).

${ }^{16} \mathrm{~F}$. Blanchette and T. P. Bigioni, "Partial coalescence of drops at liquid interfaces," Nat. Phys. 2(4), 254 (2006).

${ }^{17}$ B. Ray, G. Biswas, and A. Sharma, "Generation of secondary droplets in coalescence of a drop at a liquid-liquid interface," J. Fluid Mech. 655, 72-104 (2010).

${ }^{18}$ H. Deka, G. Biswas, K. C. Sahu, Y. Kulkarni, and A. Dalal, "Coalescence dynamics of a compound drop on a deep liquid pool," J. Fluid Mech. 866, R2-1-R2-11 (2019).

${ }^{19}$ M. Geri, B. Keshavarz, G. H. McKinley, and J. W. M. Bush, "Thermal delay of drop coalescence," J. Fluid Mech. 833, R3 (2017).

${ }^{20} \mathrm{~J}$. J. Thomson and H. F. Newall, "On the formation of vortex rings by drops falling into liquids, and some allied phenomena," Proc. R. Soc. London 39(239241), 417-436 (1886).

${ }^{21}$ E. G. Cockbain and T. S. McRoberts, "The stability of elementary emulsion drops and emulsions," J. Colloid Interface Sci. 8(4), 440-451 (1953).
${ }^{22}$ A. Gopinath and D. L. Koch, "Collision and rebound of small droplets in an incompressible continuum gas," J. Fluid Mech. 454, 145-201 (2002).

${ }^{23}$ Y. Couder, E. Fort, C. H. Gautier, and A. Boudaoud, "From bouncing to floating: Noncoalescence of drops on a fluid bath," Phys. Rev. Lett. 94(17), 177801 (2005).

${ }^{24} \mathrm{~K}$. Haldar, S. Chakraborty, and S. Chakraborty, "Influence of marangoni stress on the variation in number of coalescence cascade stages," Can. J. Chem. Eng. 97(4), 983-994 (2019).

${ }^{25} \mathrm{P}$. Dell'Aversana, J. R. Banavar, and J. Koplik, "Suppression of coalescence by shear and temperature gradients," Phys. Fluids 8(1), 15-28 (1996).

${ }^{26} \mathrm{P}$. Dell'Aversana, V. Tontodonato, and L. Carotenuto, "Suppression of coalescence and of wetting: The shape of the interstitial film," Phys. Fluids 9(9), 2475-2485 (1997).

${ }^{27}$ P. Dell'Aversana and G. P. Neitzel, “When liquids stay dry," Phys. Today 51(1), 38-41 (1998).

${ }^{28} \mathrm{R}$. Monti and R. Savino, "Correlation between experimental results and numerical solutions of the Navier-Stokes problem for noncoalescing liquid drops with Marangoni effects," Phys. Fluids 9(2), 260-262 (1997).

${ }^{29}$ R. Monti, R. Savino, and A. Cicala, "Surface tension driven-flow in noncoalescing liquid drops," Acta Astronaut. 38(12), 937-946 (1996).

${ }^{30}$ R. Savino, D. Paterna, and M. Lappa, "Marangoni flotation of liquid droplets," J. Fluid Mech. 479, 307-326 (2003).

${ }^{31}$ M. F. Schatz and G. P. Neitzel, "Experiments onthermocapillary instabilities," Annu. Rev. Fluid Mech. 33(1), 93-127 (2001).

${ }^{32}$ M. Balla, M. K. Tripathi, K. C. Sahu, G. Karapetsas, and O. K. Matar, "Nonisothermal bubble rise dynamics in a self-rewetting fluid: Three-dimensional effects," J. Fluid Mech. 858, 689-713 (2019).

${ }^{33}$ M. K. Tripathi, K. C. Sahu, G. Karapetsas, K. Sefiane, and O. K. Matar, "Non-isothermal bubble rise: Non-monotonic dependence of surface tension on temperature," J. Fluid Mech. 763, 82-108 (2015).

${ }^{34}$ G. Vazquez, E. Alvarez, and J. M. Navaza, "Surface tension of alcohol water + water from 20 to $50^{\circ} \mathrm{C}$, J J. Chem. Eng. Data 40(3), 611-614 (1995).

${ }^{35}$ V. F. Nozdrev, “The viscosities of ethanol-cyclohexane mixtures," Z. Fiz. Khim. 49, 548-549 (1975).

${ }^{36}$ I. F. Golubev, T. N. Vasil'kovskaya, and V. S. Zolin, "Experimental study of the density of aliphatic alcohols at various temperatures and pressures," J. Eng. Phys. 38(4), 399-401 (1980).

${ }^{37}$ T. Dong, W. H. Weheliye, P. Chausset, and P. Angeli, "An experimental study on the drop/interface partial coalescence with surfactants," Phys. Fluids 29(10), 102101 (2017).

${ }^{38}$ H. Ma, C. Liu, X. Li, H. Huang, and J. Dong, "Deformation characteristics and energy conversion during droplet impact on a water surface," Phys. Fluids 31(6), 062108 (2019). 